\title{
On the Symbolism of Drama Stage Character Modeling Design
}

\author{
Hui Zhang \\ Wuhan Textile University \\ Zooooooh 2005@163.com
}

\begin{abstract}
Keywords: Symbolism; Emotional needs; Drama stage character design
\end{abstract}
\begin{abstract}
With the social progress and the development of new consumption culture, people's aesthetic concept has also changed. At this time, the design advocated in the design circle is urging accordingly, and designers pay more attention to people's emotional needs. For example, the word "symbolism" has gradually emerged in the design theory. At present, "symbolism" is a very important factor in the design of characters in theater stage. This paper conducts preliminary exploration and thinking of the application of "symbolism" in the design of drama stage characters combined with examples.
\end{abstract}

\section{Introduction}

Nowadays, with the rapid development of the society, new consumption cultures are constantly emerging. In the design field, while new design concepts are being constantly absorbed, we are more determined on the consensus, i.e., more attention is paid to people's emotional needs in the process of design. The application of the "symbolism" technique, one of the important techniques of art expression, is particularly obvious in the field of design. This paper elaborates on the application of "symbolism" in drama stage character modeling design to some extent so as to carry out preliminary exploration and reflection of how to better play the supporting role of drama stage character modeling design at present.

\section{Symbolism}

Symbolism is one of the oldest and most basic way of thinking for people to learn about the world. It was produced in the mind of ancient people as a derivative of human civilization and the paleolithic rock painting was one of the best witnesses. Symbolism develops along with the development of human civilization. It can be said that all the cultural forms in the world have created and developed symbolism and its system. Ever since symbolism is rooted in human mind, it has been maintaining strong vitality and tenacity and is capricious, always accompanying around us, affecting our spiritual life and giving us unparalleled spiritual, physical and mental enjoyment.

\section{General Connotation of "Symbolism"}

"Symbolism" is a big concept that involves a wide range of disciplines and has different connotations within different disciplines. In the field of literature and art, "symbolism" also has very complex connotations. It refers to a kind of rhetoric skill, a genre, a prototype structure unit and is even regarded as a concept equivalent to the art ontology. In the Merriam Webster Dictionary, the word "symbolism" is explained as: symbolism is used to represent or imply certain things, especially an invisible thing represented by visible symbols, such as a belief, a quality, a country or a religious school. For example, the lion is the symbol of bravery, and the cross represents the Christianity. To put it simple, the so-called symbols are media (including physical objects, behaviors, rituals, language, number, structure and all the tangible and intangible things) used to express certain meanings and represent other similar things or things that are related in concept. In other words, symbolism is to give some special meaning to certain specific media. There may be some form of physical connection between these concrete images and what it is labeled, or there is no direct link. It probably comes from the myths and legends, religious stories. Or it is established 
like the Chinese idiom stories and have been passed down from generations. Not all people know where it comes from, but all the people know what it means.

\section{Features of Symbolism}

Symbolism features: First, the symbolic image represents the symbolic object, and the part can represent the whole. For example, HuaBiao can symbolize Tiananmen, Tiananmen can symbolize Beijing, and Beijing can symbolize People's Republic of China; Characteristic two is image, can represent the concept. For example, the sickle and hammer represent the peasantry and the working class. If a symbol is placed in a series of terms which have similar relations and different relations with it. Such as: logos, images, names, instructions, etc., it is easy to see that they all provide us with the relationship between the two relations. Therefore, to separate the symbol from the middle, we have to turn to other qualities of it.

As far as thinking is concerned, symbolism is a psychological phenomenon. The symbolic thinking mode is that the subject makes a comparison between the two symbols according to some connection between the symbol and the object. A mental process in which objects and abstractions are grasped by imagination and emotional experience. The creation of art is actually giving symbolic representation of what is difficult to solve in actual action. The Symbolism is not only the product of nature, but also the value of culture. Therefore, symbolism is not merely a skill, but in a sense, it is ontological. It is the mode of human thinking and the means of human communication. It is not only the symbol transmission of human emotion expression, but also the structure and knowledge system of human intelligence.

\section{"Symbolism" in the Field of Design}

The charm of symbolism in ancient civilization is that it is widely used in different cultural traditions, such as art, religion, ritual and mythology. Symbols, inherent in their irreplaceable nature, still appear frequently in art, literature, movies, and children's favorite stories. However, a large amount of advertisements, which are full of modern life, are subconsciously used in a variety of symbolic imagery languages.

As we try to introduce the general symbolic theory into the design field, something peculiar to the design domain must be incorporated into it. No matter what it is, in the design, it can be a clear theme, thought or a vague emotion, emotion, or even complex which cannot or need not be explained accurately. A work of art is a symbol, and people usually grasp it from the whole. However, the symbolic meanings of works of art, especially those of symbolic artists, are often mysterious and profound, and sometimes even the author is not clear about the implications. What they seek is detachment from worldly spirit and emotion.

\section{"Symbolism" in Drama Stage Character Modeling}

\section{Necessity of Introducing the Concept of "Symbolism" in Drama Character Modeling}

The shape of art design is the means of artistic expression. Any art need to use other means to reveal the image and artistic spirit, artistic conception, aesthetic... "The concept of styling refers to: occupy a certain space, constitute a beautiful image, make people appreciate art through vision, including painting, sculpture, architecture, etc., also known as art." Artists who unite sublimated into a point of creatures in the nature with the brush, color, in different poses and with different expressions, clay and other tools, create a beautiful life, and to make people to enjoy the beauty of art through the vision.

Here the characters are designed in a narrow sense. Narrow sense is relative broad sense. The narrow sense character modeling refers to the use of modeling methods to express them in order to express the specific intrinsic features of characters in texts. It is outside the character individual, and takes the costume, make-up and hairstyle as the intermediary, and has the function of externalization the text thought. The broad sense also includes actors' actions, speech, tone and so 
on. Drama form is the decisive factor of the form design of drama characters. The schools of drama draw on each other, and many of them cannot be simply divided into categories. The introduction of symbolic concept is aimed at more clearly analyzing the nature of various types of dramatic figure design, and more appropriately applying this stage visual factor.

\section{Application of Symbolism Form in Drama Stage Character Modeling}

Symbolic Images Represent Symbolic Objects, Parts Can Represent the Whole. In philosophy, the concepts of individual and general, part and whole, phenomenon and essence, cause and effect. No matter how clear the explanation is, in the actual process of character modeling, all these concepts must be changed into a set of effective creative mechanism. One of the keys to this mechanism is selection and elimination. In actual creation, a good design should not only focus on the surface sense of reality, but also select the most representative part from a large amount of material. Because the essence of human attention is that it is selective, and we can grasp some of the things in the horizon. But we can never master all of them. The ability of human memory is not perfect. In order to overcome this imperfection, we have mastered the means "symbolism". So people only need to provide enough for the part they can identify. Sometimes it just takes a small part. Therefore, a symbolic image has emerged, representing the symbolic object, and the part can represent the whole.

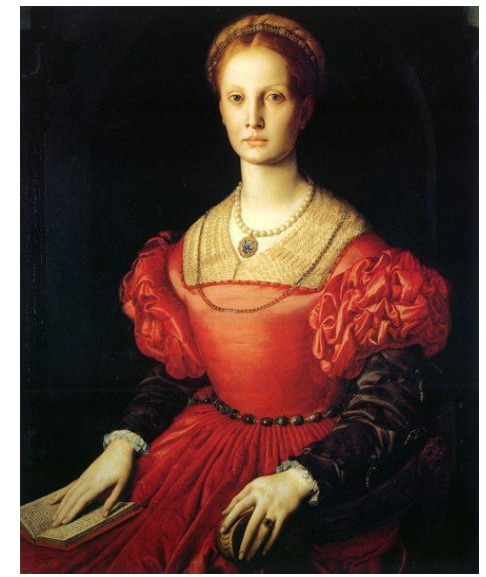

(Figure 4) Renaissance Costume

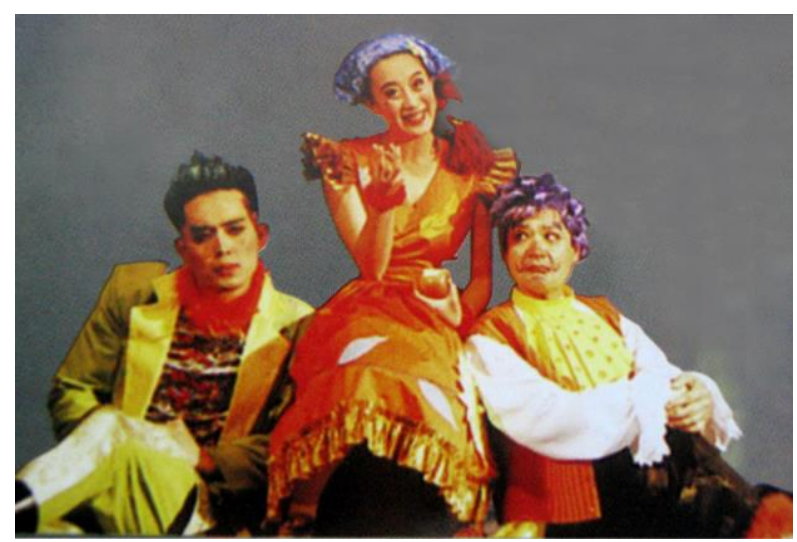

(Figure 5) Twelfth Night(2)

For example, the "Twelfth Night" (as shown in Fig.5) highlights the figures: all the women's dresses retain the outstanding features of the dresses during the Renaissance period: skirt mopping, bubble sleeves, beam waists and low cut. All the insignificant details, such as decorative lace, patters and accessories showing identity and social status are neglected. The designer shows the figures and the social and historical background in a poetic way, and also uses the alienation effect. This design pattern is both realistic and highly abstract and condensed. The figures, which are only "similar" but not "are" and the pure form makes audiences feel relaxed and pleased, which is in line with the entire humorous and beautiful love comedy. This is the most representative part retained after the removal of all unnecessary visual elements, thereby giving the audience aesthetic pleasure.

The parts represent the whole, and the figures only need to provide the part that people can identify. It shows the typical overall contour and achieves the prompt effect. High generality is the figure characteristic of narrative drama characters. The pursuit of "true vision" is a blow to stage vision.

Images Represent Concepts

From the historical development of drama, we can see that the drama always occupies an

From http://www.tucoo.com/art/renaissant_04/html/image19.htm

2) From Shanghai Lyceum Theatre's play "Twelfth Night" in 1993 
important position. But the drama between it and later is more poetic, that is, the drama form guided by the freehand brushwork. Freehand brushwork is a painting technique in Chinese painting. It refers to the charm of the object by means of succinct generalizations. The freehand brushwork in character modeling refers to the time when a certain image is more symbolic than life. By means of symbolism, analogy and abstraction, the real image is more poetic, focusing on portraying the inner character and paying more attention to the form.

From the primitive drama form to various modern drama, such as symbolism, expressionism and other drama genres, the figure of freehand brushwork is the main rhetorical model. The enjoyable form of dramatic characters refers to the shapes, elements and combinations of the characters on the stage as a metaphor. Abstract concepts such as ideas, senses, feelings, thoughts, emotions are related to emotional logic. Abstract thoughts and emotions, which require designers to use concrete modeling elements to show the intrinsic features, character modeling is the meaning of the characters want to imply, such as hope, death, depression and so on. Due to the need to show invisible things, so the impressionistic creative mechanism adopts abstract thinking mode. Freehand brushwork in the form of characters often conflict with the real world. The image on the stage is often beyond the audience's expectations, causing a strong shock, and then mobilize positive thinking, using the way of association to dig deeper meaning, thus entering the stage of the significance of the image of characters.

The formation of Chinese opera and the use of symbolism in characterization are everywhere. From Facebook to clothing, from color to style, there is hardly any type of drama that can be used extensively as a drama. These artistic forms express the creator's subjective interest and will, and form a unique imagery. The opera performance in Facebook has been stylized, all the characters have their own specific Facebook, to show the different personalities of the characters, with "blending appraise, art function of good and evil", so it is called the "picture of soul".

For different audiences, the same shape will have different meanings. Not only full attention is needed, but the dramas also rely on corresponding cultural background. To color as an example, the use of color common meaning of emotional expression is extremely common trick, designers often put a white suit to the integrity of the handsome actor, blue and white twill tie to shrewd stockbroker, the firefighter clothing like the grass green color to crazy neurotic, but if female the protagonist has enough enthusiasm and vitality, so she will have a red skirt. Although such usage is often effective, there are differences in the connotative meaning of colors in different cultural background. For example, red usually symbolizes bloodshed and violence in Western cultures, so when Westerners see the red happy scenes on the stage of China, they will have ambiguity; and Chinese audiences will also very strange seeing that white flag and white dresses are used in the wedding of "Turandot" (as shown in Fig.28; and the Chinese figure is as shown in Fig.27).

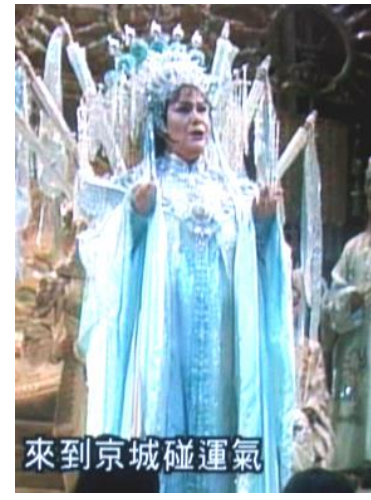

(Figure 27) "Turandot" Shot by China

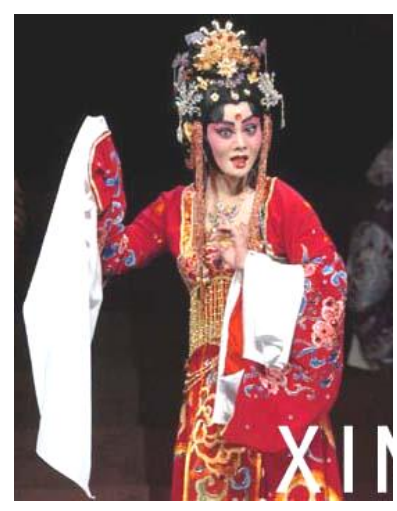

(Figure28) “Turandot” Shot by Western Countries

\footnotetext{
From "Turandot" shot by China

From "Turandot" shot by Western countries
} 
Another, the ferocious tattoos on body are originally used to symbolize strength and courage, but nowadays, since this set of techniques are frequently used in film and television drama to shape the gang leader and other villains, the meaning of tattoo has already changed and tattoos seem to be usually related to negative characters. Later, the meaning of tattoos evolved: since tattoos were branded as bad people, they were discriminated against. Therefore, it can be used to indicate they are deviant, so in the modern city, the tattoo is often used by young people to express rebellion.

Although the colors of Beijing opera masks are different in type, they are not absolute, and they also have many flexibility. For example, in the "Three Kingdoms", red is used to express the character of Guan Yu; but in the "Famen Temple", red is also used to exaggerate the color of the eunuch Liu Jin's skin and his pampered right and huge power.

Image represents the concept. Both objects of specific shape and color suggest a more profound connotation through the external form so as to better arouse audiences' enthusiasm in thinking and mine more layers that even the designers themselves have not notices.

\section{Conclusion}

Many examples of stage show prove that in the application of drama stage figure models, symbolism cannot only clearly analyze the nature of figure modeling in different types of drama so as to more appropriately reflect the unique stage visual effect, but also meet different audiences' emotional demands. In the diversified modern society, innovation and seeking difference are humans' eternal goal of pursuit. The constant creation and renewal of symbols embodies the progress and vitality of the society, but over frequent changes and excessive provision can also cause disorder and confusion in meaning, value and norm. Because not all of the drama design should be designed with symbolism, and some of the drama content, form and style are of inappropriate use. If they are randomly applied, it does not meet the artistic aesthetic and aesthetic rules. Therefore, designers are required to fully understand the script, plot, theme, social background, customs, character, performance and feasibility of the story, and stage design to coordinate with various departments before choosing whether to use symbolism and whether it can be very good for the works of drama theme service.

Therefore, correct design concepts and certain theoretical basis are the basic qualities of designers. YU Lianquan, a famous Beijing opera performing artist, once point out: "The make-up on the stage of Peking Opera is just represented a certain place." This seemingly random sentence, in fact, shows that "symbolic virtuality" is a high level of artistic creation of expression, and it is worth our lifetime experience and pursuit.

\section{References}

[1] JU Yueshi, “China's Symbolic Culture” [M], People's Press, 2001

[2] ZHAO Menglin, "Chinese Opera Faces” [M], Zhaohua Press, 2003

[3] HU Miaosheng, "Dramatic Space Full of Signs” [M] , Knowledge Press, 1985 